\title{
Force in the anal canal and anal continence
}

\author{
C. D. COllins, H. L. DUThie, T. Shelley, AND G. E. WhitTAKer \\ From the University Department of Surgery, Royal Infirmary, Sheffield
}

EDITORIAL COMMENT Radial force measurements exerted by the 'squeeze' of the anal sphincters have been recorded and it is suggested that mechanical factors rather than muscular relaxation may play an important part in the responses in the anal canal.

Explanations of the mechanism by which the anal sphincters preserve anal continence have largely been based on information gained from recordings of pressure in the anal canal and from electromyography of the levator ani and external anal sphincter muscles. The usually accepted concept of a high pressure zone in the anal canal has recently been criticized (Harris and Pope, 1964; Phillips and Edwards, 1965). In an attempt to clarify the position we have measured radial force exerted by the 'squeeze' of the anal sphincters and compared it with pressure in the anal canal in fully continent subjects.

\section{METHODS}

Seventeen male subjects, aged 21 to 70 years, without any anorectal abnormality, were studied. Pressure in the anorectum was measured using a small air-filled balloon $(5 \times 10 \mathrm{~mm}$.) (Fig. 1). A larger balloon $(5 \times 10 \mathrm{~cm}$.) was placed in the upper rectum $10 \mathrm{~cm}$. from the anal verge (rectal balloon) and was used to distend the rectum. Both balloons were attached to fine polythene tubes $(2 \mathrm{~mm}$. external diameter) which led to pressure transducers. Radial force exerted by the walls of the anal canal was measured with a 'force gauge' (Fig. 1). The outputs of the transducers were amplified and recorded on ultravioletlight-sensitive paper together with the electrical activity in the external anal sphincter recorded via a concentric needle electrode and respiratory movements from a pneumograph.

The force gauge (Fig. 2) used was similar to that described by Shelley and Warrell (1965), being essentially a single semi-conductor strain gauge bonded to one of the bars of a zeppelin-shaped cage $(3 \mathrm{~mm}$. in maximal diameter). Any radial squeeze on the cage produced a change in curvature of the bars and hence a change in resistance of the strain gauge. This resistance change was sensed by means of a Wheatstone bridge circuit, the output of which was amplified and fed to the u.v. recorder. The structure of the gauge is such that it will record the total of the forces acting to close the anal canal no matter whether these are applied uniformly radially around the gauge or come in from two sides only.

In order to assess the reproducibility of the gauge output and to compare one gauge with another the following standardization procedure was used. The gauge was placed in a Paul's tube of diameter slightly larger than that of the gauge; with the ends of the tube fixed but with the walls not stretched, an air pressure was applied around the tube thereby producing a squeeze on the gauge laying within it. Air pressure applied directly to the gauge gave no reading. Using this technique it was found that for a given pressure the output from any one gauge was reproducible to within $10 \%$ whatever the axial orientation of the gauge within the tube. By this same technique it was possible to set the gain of the amplifier to produce similar signals from different force gauges of similar outer dimensions subjected to a similar squeeze. The output characteristics of a gauge when standardized in this manner are shown in the appendix.

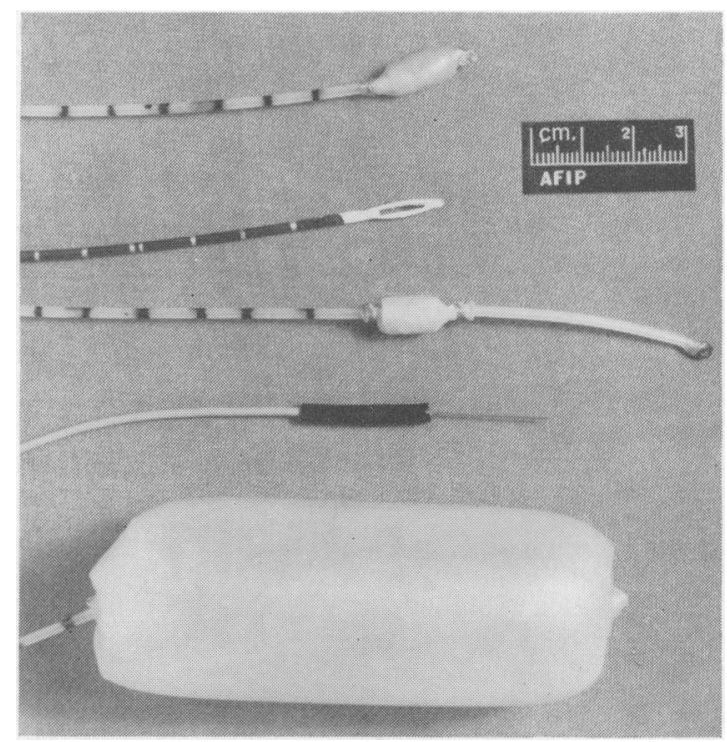

FIG. 1. Recording apparatus used (from above down). Two small air-filled balloons, one attached to the tip and the other $5 \mathrm{~cm}$. from the tip of fine polythene tubes; the force gauge, a concentric needle electrode, and the large balloon for distending the rectum. 


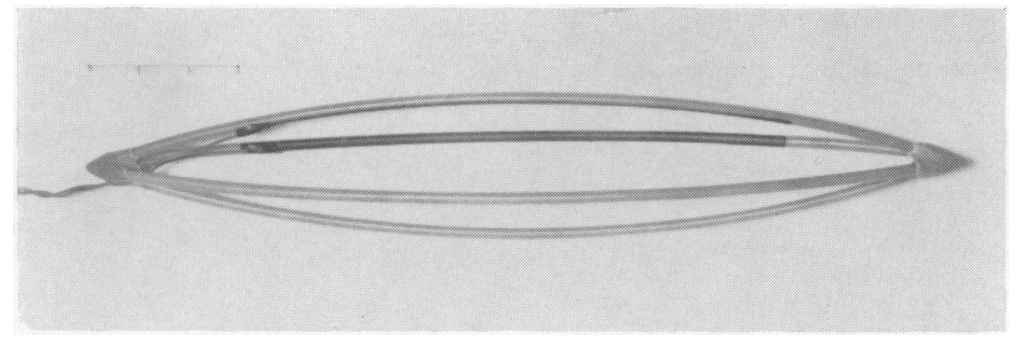

FIG. 2. A model of the force gauge to show the four curved bars joined together at their ends.

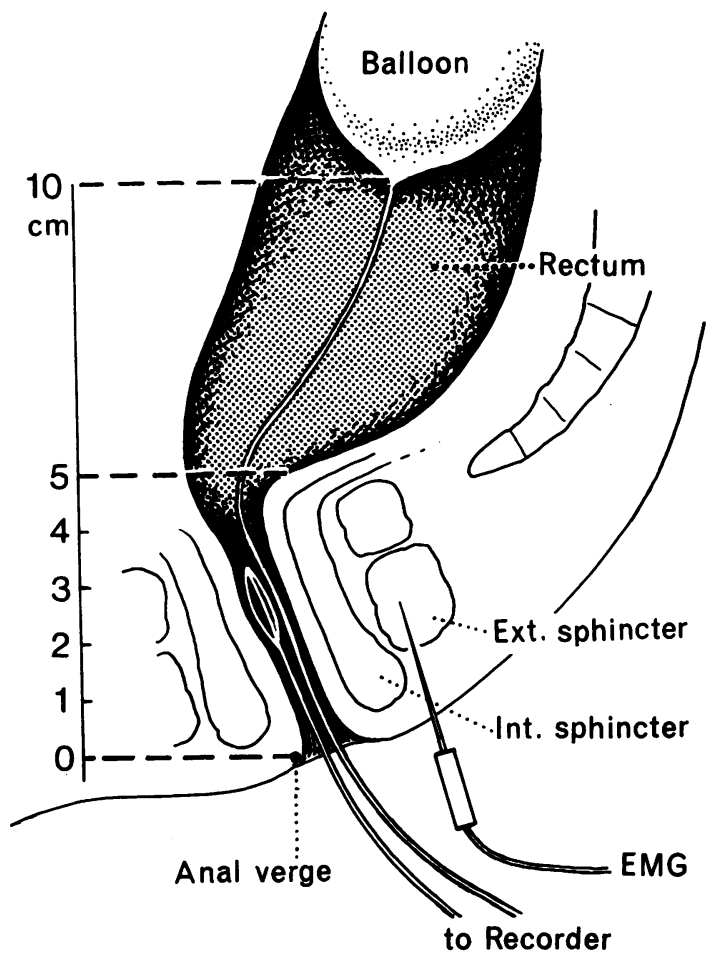

FIG. 3. Diagram of the recording units in situ for measuring force. A small balloon is substituted for the force gauge in the anal canal when pressure measurements are taken.

PROCEDURE

The subject lay on his left side and through a sigmoidoscope the rectal balloon was placed with its lower edge $10 \mathrm{~cm}$. from the anal verge (Fig. 3). Measurements were made at intervals of $1 \mathrm{~cm}$. from $5 \mathrm{~cm}$. to $1 \mathrm{~cm}$. from the anal verge with the subject at rest, and before, during, and after the following manoeuvres:

COUGHING

VALSALVA MANOEUVRE The recording devices were placed at the appropriate level and then the subject was instructed to blow into a mouthpiece to maintain a manometric pressure of $50 \mathrm{~mm}$. $\mathrm{Hg}$ for 5 to 20 seconds. A recording was made of the pressure in the manometer and in some cases intrarectal pressure was recorded with a small air-filled balloon $(5 \times 10 \mathrm{~mm}$.) or a water-filled, open-ended fine polythene tube ( $2 \mathrm{~mm}$. external diameter). The intrarectal pressure closely followed the pressure engendered in the manometer.

DISTENSION OF THE RECTUM The upper rectum was distended by inflating the rectal balloon with $100 \mathrm{ml}$. air.

VOLUNTARY SQUEEZE The patient was asked to contract the muscles in the anal region. Various phrases were used to convey the idea and also to try to forestall a generalized contraction of the abdominal muscles.

INTERPRETATION OF RECORDS In many subjects the force gauge revealed spontaneous fluctuations in the radial forces acting and these were particularly noticeable at 3 and $2 \mathrm{~cm}$. When these fluctuations were evident on the record the reading was taken as the average value about which the fluctuations occurred. Any sudden spikes on the record were ignored and only sustained levels were measured. Variability was not present on all tracings and when present was of a sufficiently regular pattern to permit drawing of a mean value.

\section{RESULTS}

AT REST As has been shown previously (Hill, Kelley, Schlegel, and Code, 1960; Duthie and Bennett, 1963), an increase in pressure above the level in the rectum was detected when the balloon was pulled into the anal canal. The withdrawal was in steps of $1 \mathrm{~cm}$. and in 14 subjects a definite and maintained increase was found on moving from 5 to $4 \mathrm{~cm}$. from the anal verge indicating that the anal canal was 4 to $5 \mathrm{~cm}$. long. In the remaining three subjects the anal canal was 3 to $4 \mathrm{~cm}$. long. The maximum pressure was at $2 \mathrm{~cm}$. from the anal verge in all but four subjects when it was at $1 \mathrm{~cm}$. from the anal verge. The mean results are shown in Fig. 4 and Table $I$.

Force was also maximal at $2 \mathrm{~cm}$. in all but five subjects when it was at $1 \mathrm{~cm}$. from the anal verge. The individual profiles were similar and the mean results were broadly similar to that of pressure. With the patient quietly at rest the maximal output from the force gauge at $2 \mathrm{~cm}$. was 77 millivolts. Mechanical considerations suggest (see appendix) that this out- 
TABLE I

Force ( $m v$.)

$\mathrm{Cm}$. from ana

verge

Resting

Rectal distension

Valsalva

Voluntary squeeze

$8.5 \pm 0.8(17)^{1}$

$10 \cdot 4+2.0(14)$

$11.6 \pm 1.9(14)$

$15.7 \pm 2.9(14)$
4

$14 \cdot 5 \pm 1 \cdot 2(17)$

$13.6 \pm 2.1(14)$

$19.1 \pm 1.9(16)$

$25.9 \pm 3 \cdot 3(15)$
3

$27 \cdot 4 \pm 3 \cdot 2(17)$
$27 \cdot 7 \pm 2 \cdot 2(14)$
$36 \cdot 2 \pm 4 \cdot 4(17)$
$46 \cdot 0 \pm 3 \cdot 4(16)$
$+0 \cdot 3 \pm 3 \cdot 1(P>0.9)$

$+8.8 \pm 5.3(\mathrm{P}<0.2)$

$+18.6 \pm 4.5(P<0.001)$
2

$35 \cdot 6 \pm 5 \cdot 0(17)$

$33.0 \pm 4.7(14)$

$49.4 \pm 5.8(16)$

$68.7 \pm 8.6(15)$
1

$34 \cdot 1 \pm 4 \cdot 5(17)$

$33.5 \pm 4.5(13)$

$47.7 \pm 4.9$ (15)

$71.0 \pm 6.9$ (12)
$-2.6 \pm 6.8(\mathrm{P}<0.6)$

$+13.8+7.4(P<0.1)$

$+33.1 \pm 9 \cdot 3(\mathbf{P}<0.01)$
$-0.6 \pm 6.1(P>0.9)$

$+13.6+6.4(\mathrm{P}<0.05)$

$+36.9 \pm 7.6(P<0.001)$

Pressure (mm. $\mathrm{Hg}$ )

Cm. from

anal verge

Resting

5

$\begin{array}{ll}\text { Valsalva } & 14.2 \pm 2.6 \pm 4.3(15)\end{array}$

Voluntary squeeze $\quad 28 \cdot 3 \pm 2 \cdot 8(14)$

4

$21 \cdot 3 \pm 2 \cdot 5(17)$

$9.9 \pm 1.8(15)$

$62 \cdot 4 \pm 4 \cdot 3(17)$

$50.9 \pm 3.8(16)$
3

$42 \cdot 2 \pm 4 \cdot 7(17)$

$17 \cdot 4 \pm 2 \cdot 3(14)$

$78 \cdot 5 \pm 6.7(17)$

$70.9 \pm 6.6(14)$
2

$56 \cdot 1+7 \cdot 2(17)$

$25 \cdot 8+3.9(12)$

$85 \cdot 1 \pm 9 \cdot 3(15)$

$102 \cdot 4 \pm 10.9(14)$
1

$41.9 \pm 6.6(17)$

$28.9 \pm 4 \cdot 1$ (12)

$67 \cdot 7 \pm 8 \cdot 7(15)$

$83 \cdot 1+7 \cdot 3(15)$

Change from

resting on:

Rectal distension $\quad+6.1 \pm 2.2(P<0.01) \quad-11.4 \pm 3.1(P<0.001) \quad-24.8 \pm 5.4(P<0.001) \quad-30.3 \pm 8.5(P<0.01)$

Valsalva

$+6.1 \pm 2.2(\mathrm{P}<0.01)$
$+37.5 \pm 4.1(\mathrm{P}<0.001)$

$+41 \cdot 1 \pm 4.8(\mathrm{P}<0.001)$
$+29.6 \pm 4.8(\mathrm{P}<0.001)$

$-24.8 \pm 5.4(\mathrm{P}<0.001)$
$+36.3 \pm 7.9(\mathrm{P}<0.001)$

Voluntary squeeze $+20.2 \pm 2.6(P<0.001)$

$+28.7 \pm 7.6(\mathrm{P}<0.001)$

$+29.0 \pm 10.6(\mathrm{P}<0.02)$
$+46.3 \pm 12.4(\mathrm{P}<0.001)$

$-13.0+7.9(P<0.2)$

$+25.8 \pm 10.5(\mathrm{P}<0.05)$

$+41.2 \pm 9.5(\mathrm{P}<0.001)$

Mean values \pm S.E.M. $\quad{ }^{1}$ Numbers of subjects in brackets. $\quad$ 'Significance of $t$ test in brackets.

put was approximately equivalent to 14 grams weight. It seems reasonable therefore to assume that, in general, the radial forces needed for closure under these conditions are of less than this value. While the recordings of force were reproducible on repeated readings in the same subject, a wide range was observed over the series, for example at $2 \mathrm{~cm}$. force gauge, output ranged from 16 to 77 millivolts with a mean of $36 \mathrm{mV} . \pm 5.0$ S.E.M.

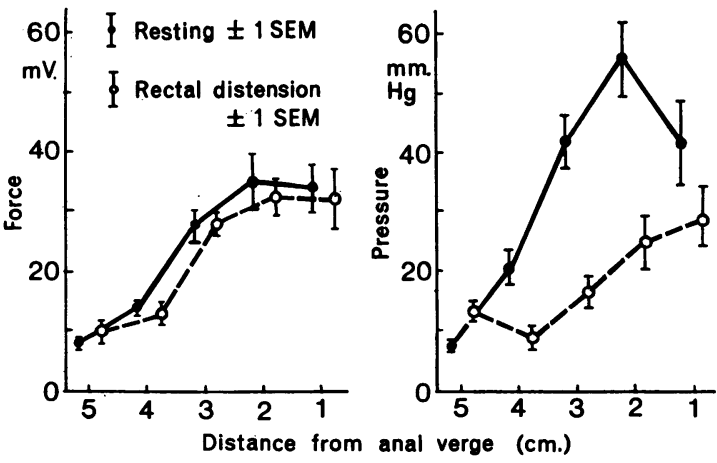

FIG. 4. Mean pressure and force in the anal canal recorded via a terminal air-filled balloon $(5 \times 10 \mathrm{~mm}$.) and the force gauge in 17 patients, showing values under resting conditions and on rectal distension.
COUGHING A simultaneous increase in pressure, force, and electrical activity was noted in all subjects on coughing (Fig. 5).

VALSALVA MANOeuvre During the Valsalva manoeuvre intraabdominal pressure increased to 45 to $50 \mathrm{~mm} . \mathrm{Hg}$ and a corresponding increase in pressure was found in the anal canal at $4 \mathrm{~cm}$. and $3 \mathrm{~cm}$. Nearer the anal verge the increment was smaller and less significant. The same results were obtained when the balloon was attached $5 \mathrm{~cm}$. from the tip of the recording tube.

The squeeze applied to the force gauge by the walls of the anal canal showed a slight increase over resting levels which was much smaller than the increment in pressure. The changes were also different in that no large increase was seen in the upper part of the anal canal. The readings with the force gauge at $1 \mathrm{~cm}$. were unreliable during a Valsalva manouevre as the gauge tended to slip out.

Electrical activity in the external anal sphincter showed a moderate increase during the Valsalva manoeuvre (Fig. 5).

RECTAL DISTENSION On inflating the rectal balloon with $100 \mathrm{ml}$. air the pressure in the anal canal decreased promptly and reached a nadir in five to 10 seconds (Fig. 7). The mean changes were statistically significant (Fig. 4, Table I). In about half the tests a slow increase in pressure followed, to reach a plateau 10 to $20 \mathrm{~mm}$. $\mathrm{Hg}$ below the resting 

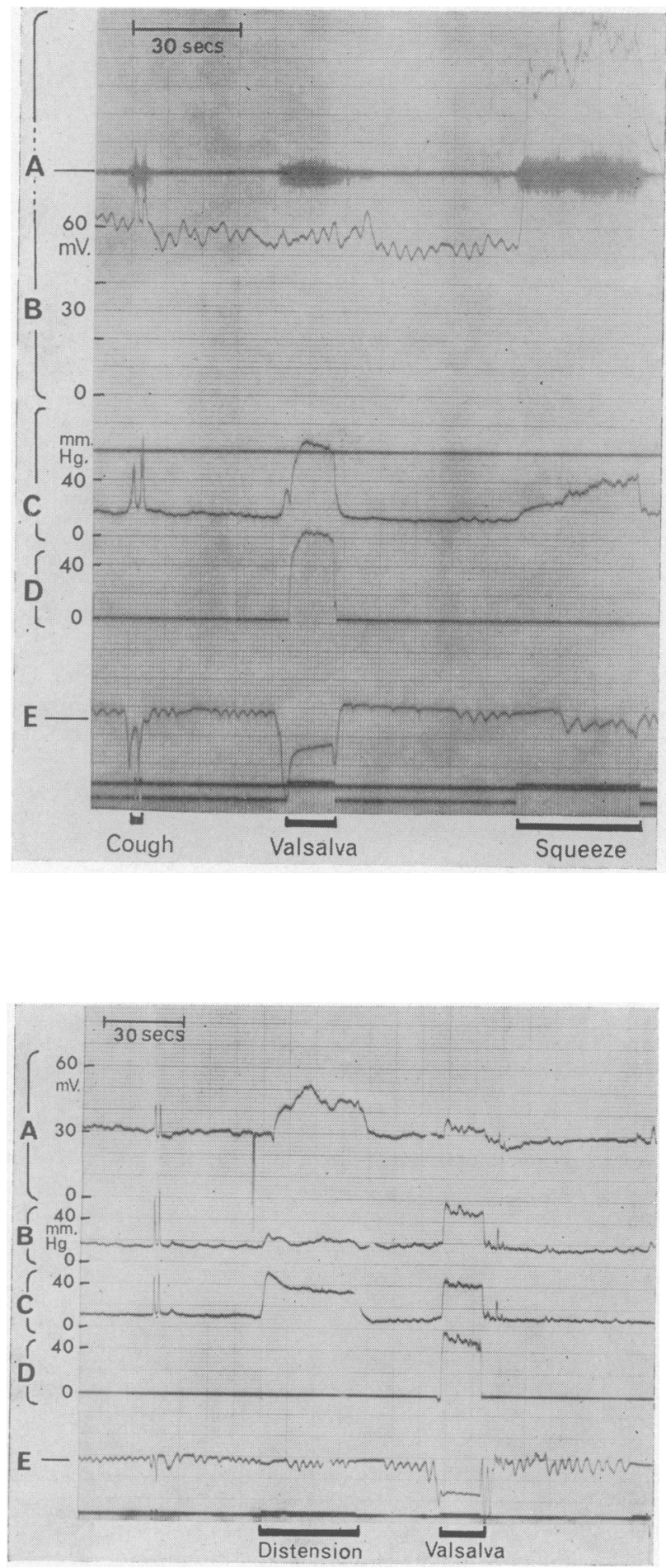

FIG. 5. Simultaneous tracings (from above down) of $(A)$ electrical activity in the external anal sphincter, $(B)$ force from the force gauge at $2 \mathrm{~cm}$. from the anal verge, $(C)$ pressure from the small balloon at $4 \mathrm{~cm}$., (D) expiration pressure during Valsalva manoeuvre, $(E)$ pneumograph of respiratory movements. $A$ simultaneous increase in force, pressure, and electrical activity is seen on coughing. On Valsalva manoeuvre there is little change in the force but a marked increase in pressure and electrical activity. On voluntary squeeze there is a marked increase in force and electrical activity with a small increase in pressure. It must be noted that the force gauge is $2 \mathrm{~cm}$. distal to the small balloon.

FIG. 6. Simultaneous tracings (from above down) of $(A)$ force at $3 \mathrm{~cm}$. from the anal verge, $(B)$ pressure from the small balloon at $5 \mathrm{~cm}$. just within the rectal ampulla, $(C)$ pressure from a small balloon placed alongside the distending rectal balloon, (D) expiratory pressure during Valsalva manoeuvre, $(E)$ pneumograph of respiratory movements. An increase in force can be seen at the time of rectal distension. Valsalva manoeuvre is accompanied by a small increase in force in the anal canal. 


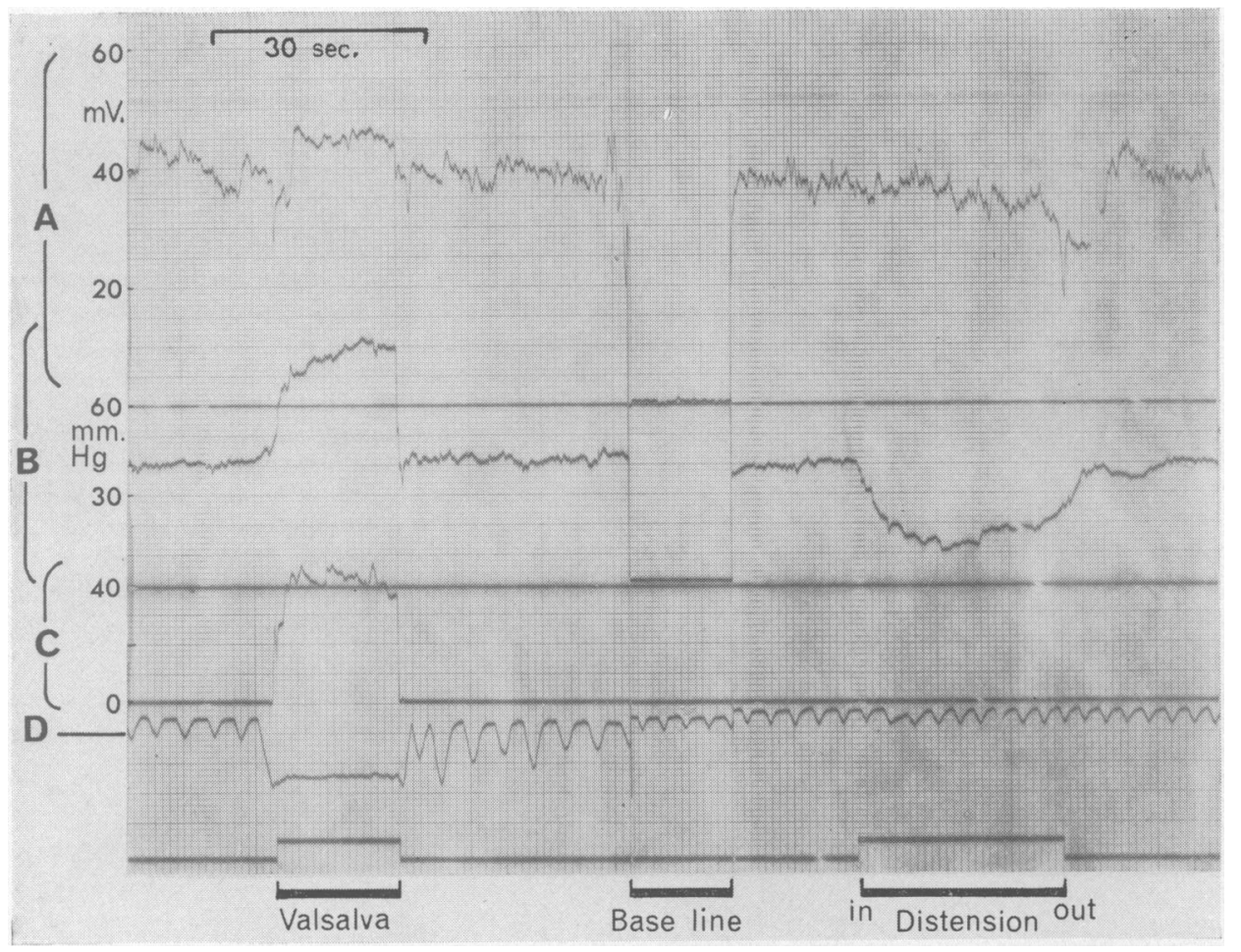

FIG. 7. Simultaneous tracings (from above down) of $(A)$ force at $2 \mathrm{~cm}$. in anal canal, (B) pressure in anal canal at $3 \mathrm{~cm}$., $(C)$ expiratory pressure during Valsalva manoeuvre, $(D)$ pneumograph of respiratory movements. Valsalva is accompanied by a large increase in pressure in the anal canal and a small increase in force. Rectal distension is associated with a definite decrease in pressure and a slight fall in force in the anal canal.

level, while the rectal distension was still maintained. The recordings at $5 \mathrm{~cm}$. represented the accommodated intrarectal pressure.

In contrast to this prompt reduction in pressure, force in the anal canal showed a variable response during distension of the rectum: an increase (Fig. 6), a decrease (Fig. 7), or no change might be observed. For example, at $2 \mathrm{~cm}$. from the anal verge, an increase was seen in five subjects, a decrease in four subjects, and no change in the remainder. The mean results showed no significant response (Fig. 4, Table I).

Electrical activity in the external anal sphincter increased transiently both on inflation and on deflation of the balloon. Otherwise it remained at resting levels. No inhibition of the electromyogram was observed at this level of rectal distension: it required inflation of the rectal balloon with 250 to $300 \mathrm{ml}$. of air to produce inhibition.

VOLUNTARY SQUEEZE Voluntary contraction of the muscles in the anal region was accompanied by a significant increase in pressure and radial force in the anal canal and in electrical activity in the external anal sphincter (Fig. 5). The changes in pressure and radial force were greatest in the lower part of the anal canal at $2 \mathrm{~cm}$. and $1 \mathrm{~cm}$. where the external anal sphincter might be expected to exert its maximal effect. The increase in pressure and force at $5 \mathrm{~cm}$. from the anal verge, i.e., in the lower part of the rectal ampulla, show that we were not entirely successful in limiting the voluntary squeeze to muscles in the perineum.

\section{DISCUSSION}

Concepts of anal sphincteric action have been based hitherto on pressure measurements and some aspects of interpretation have proved difficult. Comparison of results from one group of workers to another have not been made any easier by the range of recording units used: from fine tubes (1-2 mm. diameter) (Hill et al., 1960) with end or side holes to large balloons 
(3-5 cm. diameter) (Schuster, Hendrix, and Mendeloff, 1963). The present study has concentrated on measuring the radial force in the anal canal and comparing it with pressure as seen by a small balloon. The great advantage of the force gauge is that it is not affected by intraluminal pressure. Standardization of the output of the force gauge in the calibrating device has allowed comparison of the results of one subject with another on a quantitative basis.

With the subject at rest the pressure in the anal canal showed a profile similar to that previously recorded (Hill et al., 1960; Duthie and Bennett, 1963). Force in the anal canal showed a similar distribution, and under these circumstances, it could be argued that the radial force was responsible for the pressure measured in the anal canal. This may seem to be stating the obvious, but in other circumstances, for example, during a Valsalva manoeuvre, the direct relationship did not hold. It was also noticeable that no localized high force zone was defined such as might have been expected were the muscles of the pelvic floor forming an orifice through which the anal canal could pass to be compressed laterally by intraabdominal pressure (Phillips and Edwards, 1965).

The Valsalva manoeuvre gave a reproducible increase in intraabdominal and intrarectal pressure and the latter closely followed the expiratory pressure. Pressure in the anal canal was measured with several devices, an open-tipped water-filled tube, a small balloon at the end of the recording tube or placed $5 \mathrm{~cm}$. from the tip of the tube. With each device, an increase in pressure was observed in the anal canal during the Valsalva manoeuvre, in contrast to the findings of Harris and Pope (1964). The increase in pressure found in the upper part of the anal canal is similar to that obtained by Phillips and Edwards (1965) when their subjects were 'bearing down', i.e., it seems that transmission of pressure is limited to the upper part of the anal canal. However, the measurement of radial force in the anal canal shows no evidence that intraabdominal pressure is forcing the walls of the anal canal together as these authors postulated. Such a mechanism would have shown a distinct increase in radial force at $4 \mathrm{~cm}$. and $3 \mathrm{~cm}$. from the anal verge. The increase in pressure in the anal canal at $4 \mathrm{~cm}$. and $3 \mathrm{~cm}$. from the anal verge was not accompanied by a proportionate increase in radial force in our studies. This apparent contradiction may be explained by transmission of intrarectal pressure by the mucosa and submucosa of the anal canal within the muscular tube of the anal sphincters. This would affect the pressure recording unit but would not alter the radial force exerted by the muscles.

Despite the fact that a fall in pressure in the anal canal in response to distension of the rectum has been recorded over 30 years ago (Denny-Brown and Robertson, 1935), a fully acceptable explanation is still being sought. The response was observed in normal subjects, in patients with spinal cord transection, in patients with sacral cord lesions, and more recently in patients with Hirschsprung's disease (Schuster et al., 1963). It has been difficult to identify a nervous or neuromuscular pathway for this response but the usual explanation is that the anal sphincters relax. Electromyography of the levator ani and external anal sphincter muscles has shown an inhibition of electrical activity on distension of the rectum (Porter, 1961) and this finding appeared to fit the above explanation. However, we have confirmed our previous results showing that, when 100 $\mathrm{ml}$. of air was used to distend the upper rectum, a decrease in pressure in the anal canal occurred promptly and the electrical activity of the external anal sphincters showed no diminution (Duthie and Watts, 1965). If distension were continued to the level required to produce inhibition of the electromyogram (250 to $300 \mathrm{ml}$. of air) the decrease in pressure in the anal canal had largely passed off. Another finding which was difficult to explain was the occasional recording of zero pressure along the anal canal during rectal distension without any evidence of incontinence. The failure to explain this response on neuromuscular grounds has led to suggestions that the physical characteristics of the anal canal may contribute to the pressure recorded (Phillips and Edwards, 1965; Duthie and Watts, 1965).

Our results using the force gauge would seem to lend support to a physical explanation of the decrease in pressure in the anal canal in response to distension of the rectum. As a result, muscular relaxation seems an unlikely explanation for the decrease in pressure. A slight inwards movement was frequently observed on inflation of the rectal balloon, and, although it was placed $10 \mathrm{~cm}$. from the anal verge, it may be that the stretching of the rectal walls caused the mucosa and submucosa to move relative to the muscular walls of the canal. Such a relative movement inwards could lead to a change in shape of the pressure balloon which would show as a decrease in the pressure reading. These circumstances would not significantly affect the radial forces acting and therefore would leave the force gauge reading unaltered.

A possible criticism of this physical explanation is that in patients who have had an anterior resection, distension of the rectum below the suture line gives rise to this decrease of pressure in the anal canal, whereas distension in the colon above the suture line does so only occasionally (Goligher, Duthie, Dedombal, and Watts, 1965). In these cases fibrous 
scar tissue may prevent the rectal walls being pulled up and hence diminish the effect on the anal canal.

In our explanation of the changes in pressure during Valsalva manoeuvre and during rectal distention we have postulated that pressure can be transmitted by movement of the mucosa and the submucosa of the upper part of the anal canal relative to the muscular wall. If this were the case it would help to explain the lack of transmission of pressure to the lower part of the anal canal since the mucosa and submucosa are anchored firmly to the muscular layers by the anal glands at the level of the anal valves about $2 \mathrm{~cm}$. from the anal verge.

As has been mentioned already, several anomalies in the results of pressure studies of the anorectum have diminished the acceptance of a high pressure zone in the anal canal as the mechanism of anal continence. Our use of the force gauge has demonstrated a radial force in the anal canal which is fairly constant over a wide range of intrarectal pressure and which is not subject to the big changes seen on pressure recordings. It is suggested that this force in the anal canal might provide a basis for the preservation of continence.

\section{SUMMARY}

Force and pressure in the anal canal of 17 normal subjects were measured at the same time as electrical activity in the external anal sphincter. All three parameters showed similar responses at rest, on coughing, or voluntary squeeze of the perianal muscles. On a Valsalva manoeuvre the changes in force were less than those of pressure, especially in the upper anal canal. When the rectum was distended with a balloon the well known decrease of pressure in the anal canal was not accompanied by any definite change in force. On the basis of these findings it is suggested that mechanical factors rather than muscular relaxation may play an important part in the responses in the anal canal.

\section{REFERENCES}

Denny-Brown, D., and Robertson, E. G. (1935). An investigation of the nervous control of defaecation. Brain, 58, 256-310.

Duthie, H. L., and Bennett, R. C. (1963). The relation of sensation in the anal canal to the functional anal sphincter: a possible factor in anal continence. Gut, 4, 179-182.

- and Watts, J. M. (1965). Contribution of the external anal sphincter to the pressure zone in the anal canal. Ibid., 6, 64-68.

Goligher, J. C., Duthie, H. L., Dedombal, F. T., and Watts, J. McK. (1965). Abdomino-anal pull-through excision for tumours of the mid-third of the rectum. Brit. J. Surg., 52, 323-335.

Harris, L. D., and Pope, C. E., II (1964). 'Squeeze' vs. resistance: an evaluation of the mechanism of sphincter competence. $J$. clin. Invest., 43, 2272-2278.
Hill, J. R., Kelley, M. L., Jr., Schlegel, J. F., and Code, C. F. (1960). Pressure profile of the rectum and anus of healthy persons. Dis. Colon Rect., 3, 203-209.

Phillips, S. F., and Edwards, D. A. W. (1965). Some aspects of anal continence and defaecation. Gut, 6, 396-406.

Porter, N. H. (1961). Megacolon: a physiological study. Proc. roy. Soc. Med., 54, 1043-1047.

Schuster, M. M., Hendrix, T. R., and Mendeloff, A. I. (1963). The internal anal sphincter response: manometric studies on its normal physiology, neural pathways, and alteration in bowel disorders. J. clin. Invest., 42, 196-207.

Shelley, T., and Warrell, D. W. (1965). Measurement of intra-vesical and intra-urethral pressure in normal women and in women suffering from incontinence of urine. J. Obstet. Gynaec. Brit. Cwlth, 72, 926-929.

\section{APPENDIX}

With the patient at rest and the gauge at $2 \mathrm{~cm}$. a mean output of 36 millivolts was obtained. From this value and a knowledge of the strain gauge, amplifier, and recorder sensitivity it is possible to calculate the strain in the beam. From this strain and the structure of the gauge it is possible to estimate the force which will produce this strain and output. This force is between 6 and $8 \mathrm{gwt}$. depending upon whether it is considered to be loaded at its midpoint or uniformly. This theoretical value was confirmed in two ways:

(1) Using the curve obtained from the standardizing equipment the average force acting was calculated from a knowledge of the pressure and the active area of the bar. This force was found to be $7 \mathrm{gwt}$. when an output of 36 millivolts occurred.

(2) By the application of forces to the midpoints of the bars of the gauge the force per bar required to produce an output of 36 millivolts was found to be $6.2 \mathrm{gwt}$. For other values the response characteristics of a force gauge are shown in Figure 8.

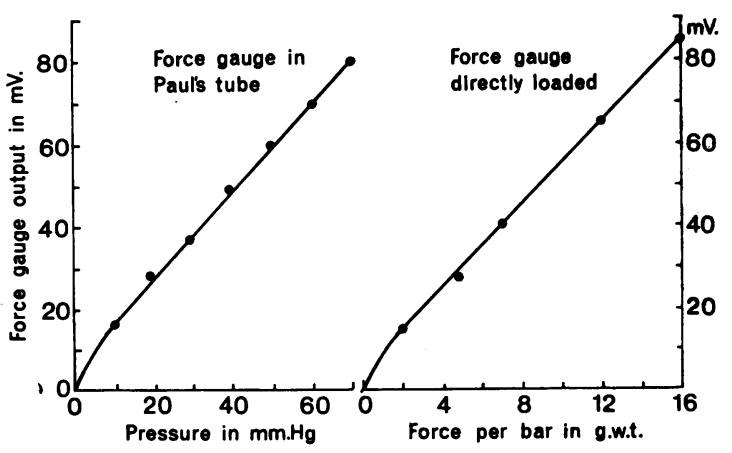

FIG. 8. Response characteristics of force gauge in the standardizing equipment (a Paul's tube) and when directly loaded.

In view of the above considerations it is thought that the radial forces monitored in the anal canal with the patient at rest is in the region of $6 \mathrm{gwt}$. 